\title{
Diseño y desarrollo de un prototipo Checking - Fixture para componentes de la industria automotriz con un enfoque hacia la industria 4.0
}

\section{Design and development of a prototype Checking - Fixture for components of the automotive industry with a focus on Industry 4.0}

\author{
PAZ-CABRERA, Mauro †*, MANDUJANO-NAVA, Arturo, LANDA-CALDERON, Rosa María y \\ MENDOZA-DERRAMADERO, José De La Cruz
}

Universidad Politécnica de Guanajuato, Departamento de Ingeniería Automotriz

ID $1^{\mathrm{er}}$ Autor: Mauro, Paz Cabrera / ORC ID: 0000-0003-0728-7377, CVU CONACYT ID: 305750

ID $1^{\mathrm{er}}$ Coautor: Arturo, Mandujano Nava / ORC ID: 0000-0003-2022-4397, CVU CONACYT ID: 270254

ID $2^{\text {do }}$ Coautor: Rosa María, Landa Calderón / ORC ID: 0000-0002-1492-9609, CVU CONACYT ID: 737914

ID $3^{\mathrm{er}}$ Coautor: José De La Cruz, Mendoza Derramadero / ORC ID: 0000-0001-6128-2660, CVU CONACYT ID: 424690

DOI: $10.35429 / J T D .2019 .12 .3 .1 .6$

Recibido: 20 de Octubre, 2018; Aceptado 10 de Diciembre, 2018

\begin{abstract}
Resumen
El presente trabajo muestra el diseño y la integración de varios complementos en un dispositivo a nivel prototipo, que lleva a cabo el seguimiento de las características físicas y dimensionales para componentes de la industria automotriz con base a las nuevas tendencias tecnológicas, como lo es la industria 4.0. Para ello se hace uso de software de diseño CAD Solidworks 2017, el empleo de sistemas CAM con el software EdgeCam 2019 como elemento de manufactura, se emplea sistema de visión utilizando como interface el software de Matlab y la herramienta estadística de Minitab 2017, todo ello integrado en un dispositivo que ayude a conocer el comportamiento de los parámetros que se definan como críticos en la producción de componentes automotrices y poder llevar a cabo los ajustes necesarios en los parámetros de producción para reducir la variabilidad en el proceso. Buscando tener en tiempo real la información y poder tomar decisiones oportunas. Generando las cartas de control estadístico correspondientes, persiguiendo con ello evitar posibles retrabajos o en su caso la generación de scrap.
\end{abstract}

Checking - Fixture, Industria 4.0, Industria Automotriz

\begin{abstract}
The present work shows the design and the integration of several complements in a device at prototype level that carries out the tracking of the physical and dimensional characteristics for components of the automotive industry based on the new technological trends, as it is the industry 4.0. For this purpose, CAD design software such as Solidworks 2017 is used, the use of CAM systems with EdgeCam 2019 software as a manufacturing element, the vision system is used using the Matlab software and the Minitab 2017 statistical tool as an interface a device that helps to know the behavior of the parameters that are defined as critical in the production of automotive components and be able to carry out the necessary adjustments in the production parameters to reduce the variability in the process. Looking to have information in real time and make timely decisions. Generating the corresponding statistical control charts, with the aim of avoiding possible reworking or in the case of scrap generation
\end{abstract}

Checking - Fixture, Industry 4.0, Automotive Industry

Citación: PAZ-CABRERA, Mauro, MANDUJANO-NAVA, Arturo, LANDA-CALDERON, Rosa María y MENDOZADERRAMADERO, José De La Cruz. Diseño y desarrollo de un prototipo Checking - Fixture para componentes de la industria automotriz con un enfoque hacia la industria 4.0. Revista del Desarrollo Tecnológico. 2019 3-12: 1-6

\footnotetext{
* Correspondencia del Autor (Correo electrónico: mpaz@upgto.edu.mx)

$\dagger$ Investigador contribuyendo como primer autor.
} 


\section{Introducción}

La carrocería de un automóvil está compuesta por diferentes partes, como lo son: un chasis, un conjunto de panel frontal, un conjunto de panel trasero, conjuntos de paneles laterales izquierdo y derecho, un techo y un conjunto de piso y cada conjunto también se divide en subconjuntos o piezas hasta niveles de pieza. Actualmente, los reemplazos de los nuevos modelos se producen cada vez más rápido, en los que la forma de la carrocería del automóvil suele ser uno de los cambios más importantes. Si se establece un sistema de fabricación correspondiente cuando se desarrolla un nuevo modelo automotriz, el costo es muy alto. Por lo tanto, muchas compañías automotrices intentan adoptar sistemas de accesorios flexibles para disminuir el costo de desarrollo de una nueva carrocería automotriz (Kuigang Yu, ShiyaWang, YananWang and Zhihong Yang, 2018).

Uno de los principales problemas objetivos de la Industria 4.0, es que se reduzcan los tiempos de preparación y cambio de producción de manera que podamos obtener una fabricación continua. Con esto, nos referimos a sistemas flexibles, integrados en la cadena de valor y sobre todo eficientes. Se busca conseguir sistemas de fabricación que permitan producir una gran variedad de productos diferentes en tiempos reservados antes a la producción en cadena (Barros Losada, 2017).

El diseño asistido por computadora por ejemplo, se ha vuelto indispensable en el mundo de la ingeniería, el poder probar la resistencia de piezas antes de su fabricación ha podido ahorrar tiempo y dinero, el saber que una pieza va a resistir o tiene que ser rediseñada antes de ser fabricada. El desarrollo de un prototipo o herramental hace uso de las herramientas de diseño y la manufactura asistida por computadora. Utilizando las diferentes combinaciones $\mathrm{CAD}, \mathrm{CAM}, \mathrm{CAD} / \mathrm{CAM}$ ha tenido un gran efecto en la manera en que se realiza la fabricación y ha permitido mejorar la precisión y confiabilidad del proceso así como de la productividad de los trabajadores (Jensen, D. Helsel, \& R. Short, 2003). Un prototipo o dispositivo debe satisfacer los requisitos de diseño, especificaciones de calidad y normas, además de ser un modelo original del diseño para evaluar la forma, la función y el ajuste o ensamble antes de iniciar la producción (Evans).
La Metrología Dimensional se encarga de analizar y desarrollar las técnicas de medición que determinan correctamente las magnitudes lineales y angulares, así como de la evaluación del acabado superficial de las piezas manufacturadas (Gonzalez Gonzalez \& Zeleny Vazquez, 1995).

Una de las principales herramientas de la Metrología Dimensional son los dispositivos Checking Fixture, el cual es utilizado para la localización, posición y ubicación específica de un punto o de una medida requerida, estos son una necesidad para la industria, permitiendo optimizar su productividad y conformidad de tal forma de asegurar su calidad, así como, la capacidad de intercambio agilizando el trabajo de la máquina y el trabajador.

El control de calidad es un conjunto de actividades orientadas al cumplimiento de los requisitos de calidad que se apoya de técnicas estadísticas para dar origen al Control Estadístico de la Calidad.

Una de las tareas clave del Control Estadístico de un proceso será conocer su variabilidad ocasionada por los materiales, máquinas, mano de obra, mediciones, medio ambiente y el método.

El pensamiento Estadístico se considera una filosofía de aprendizaje y acción que establece la necesidad de un análisis adecuado de los datos de un proceso, como una acción indispensable para mejorar su calidad y reducir su variabilidad (Gutierrez Pulido, 2013).

El propósito de cualquier análisis de un sistema de medición deberá ser: entender mejor las fuentes de variación que pueden influenciar los resultados producidos por el sistema.

El propósito de realizar un análisis del sistema de medición es asegurar que la información recolectada sea una representación real de lo que está ocurriendo en el proceso.

\section{Planteamiento del Problema}

Debido al desarrollo industrial a nivel global y en específico en la industria automotriz, es importante que en nuestro país se lleve a cabo una propuesta adecuada a dicho crecimiento. 
En el estado de Guanajuato y estados vecinos se ha desarrollado un cluster automotriz que busca la competitividad y el crecimiento sustentable de la región; hoy en día en el campo de la metrología la mayoría de los sistemas de medición conocidos como Gages son en su gran mayoría analógicos, por lo que la propuesta que se presenta va enfocada a que dichos elementos de medición migren hacia sistemas automáticos, empleando para ello la visión en tiempo real y transformar dichas imágenes en datos cuantitativos para la obtención de datos estadísticos, con el objetivo de mantener un proceso mejor controlado.

\section{Metodología}

Con base a lo anterior, se propone la metodología que se muestra en la figura 1 para el diseño de Checking Fixtures para la industria automotriz, la cual busca establecer un procedimiento confiable para el desarrollo de proyectos de medición donde los análisis estadísticos de R\&R brinden un comportamiento uniforme entre lecturas de las características a evaluar y con ello poder cumplir con los parámetros que se definan como especificaciones del cliente y sus tolerancias, tanto geométricas como dimensionales.

\begin{tabular}{|c|}
\hline 1. Investigacion Preliminar de la parte \\
\hline 2. Diseño y especificacion de la parte. \\
\hline $\begin{array}{l}\text { 3. Definicion de los requerimientos del } \\
\text { Sistema. }\end{array}$ \\
\hline $\begin{array}{l}\text { 4. Diseño de componentes y contruccìon de } \\
\text { Prototipo 3D. }\end{array}$ \\
\hline 5. Evaluacion y mejora de Prototipo 3D. \\
\hline $\begin{array}{l}\text { 6. Diseño tecnico de componentes } \\
\text { contruccion de Prototipo Checking Fixture. }\end{array}$ \\
\hline $\begin{array}{l}\text { 7. Programacion y prueba Prototipo Checking } \\
\text { Fixture. }\end{array}$ \\
\hline $\begin{array}{l}\text { 8. Analisis SPC y Gage R\&R Prototipo } \\
\text { Checking Fixture. }\end{array}$ \\
\hline $\begin{array}{l}\text { 9. Operacion y control de Prototipo Checking } \\
\text { Fixture. }\end{array}$ \\
\hline
\end{tabular}

Figura 1 Metodología para Diseño y desarrollo de Checking Fixture

Fuente: Elaboración Propia

\section{Desarrollo}

Para dar comienzo con el proceso de diseño y construcción es importante conocer la pieza a evaluar y los requerimientos que se deben de cumplir para que la pieza sea funcional en el vehículo y con ello poder establecer los elementos datum que servirán como referencia para la construcción de los gages con los cuales se van a evaluar las piezas. Ver pieza muestra en la Figura 2.

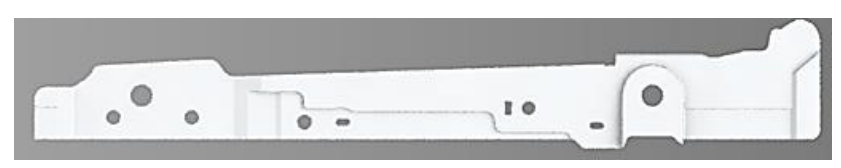

Figura 2 Dibujo de pieza de referencia para análisis Fuente: Elaboración Propia

Una vez que se pudo llevar a cabo la identificación de la pieza y su funcionalidad, es importante caracterizar la pieza por lo que para ello, se lleva a cabo un dibujo técnico donde se especifiquen sus dimensiones, datums y sus tolerancias tanto geométricas como dimensionales, ver figura 3 .

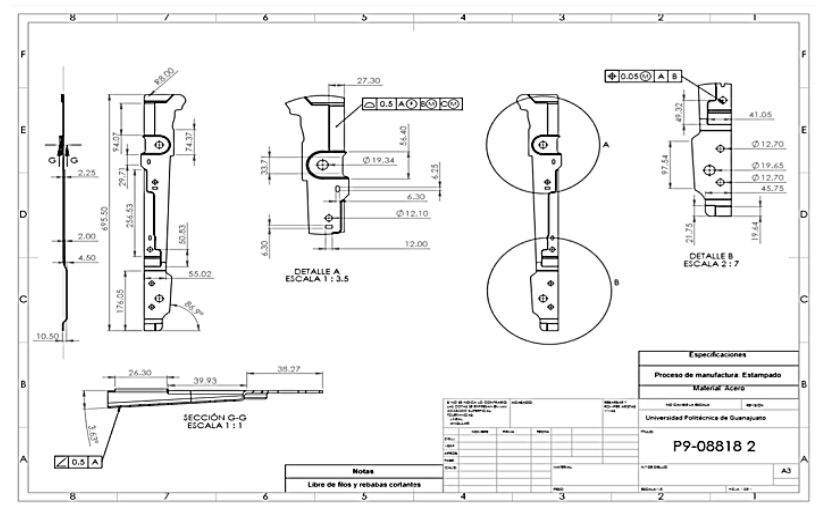

Figura 3 Diseño mediante ingeniería inversa Fuente: Elaboración Propia

Una vez parametrizada la pieza se lleva a cabo la generación de la propuesta y/o propuestas del gages que cumpla con los elementos de evaluación adecuados para la pieza, con lo cual se lleva a cabo el diseño del prototipo virtual en software de CAD Solidworks 2017, teniendo en cuenta de que el dispositivo debe tener la capacidad de identificar y evaluar las características importantes de la pieza y su relación en cuanto a su posición espacial y funcional en el vehículo, ver figuras 4 y 5 . 


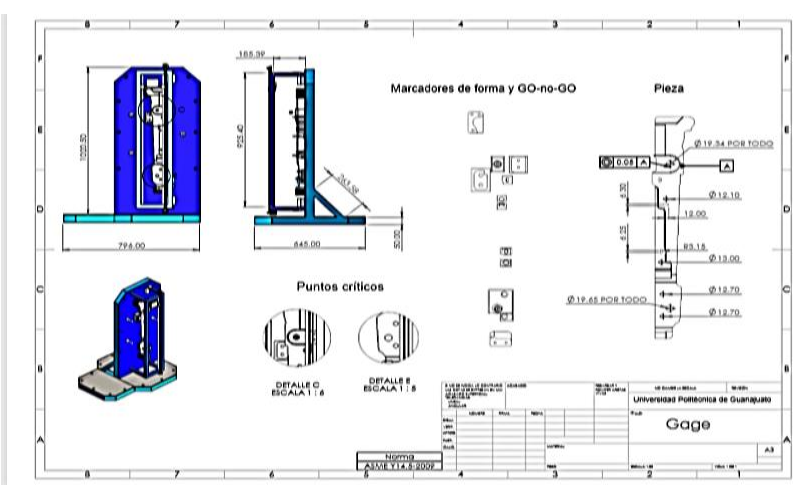

Figura 4 Diseño de Gage empleando herramientas de CAD

Fuente: Elaboración Propia

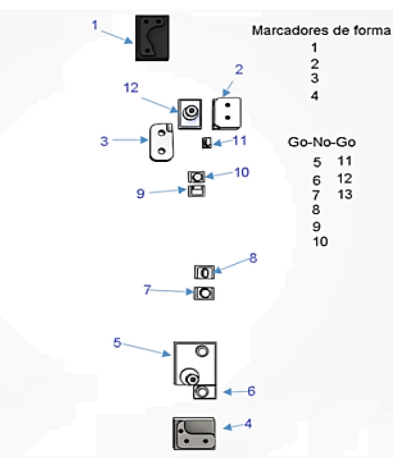

Figura 5 Elementos que ayudan a evaluar en el Gage las características importantes

Fuente: Elaboración Propia

Una vez validado el diseño de CAD, se procedió a realizar la fabricación y ensamble del gage con ayuda de las herramientas de CAM y un centro de maquinado CNC. La construcción física del gage es importante, ya que permite realizar las pruebas correspondientes directamente sobre la pieza a evaluar. En la figura 6 se muestra una vista frontal del prototipo del gage que se fabricó, el cual contiene un mecanismo con un sistema de visión que desliza sobre dos guías lineales las cuales permiten hacer el barrido y el procesamiento de imágenes en tiempo real. Ver Figura 6.

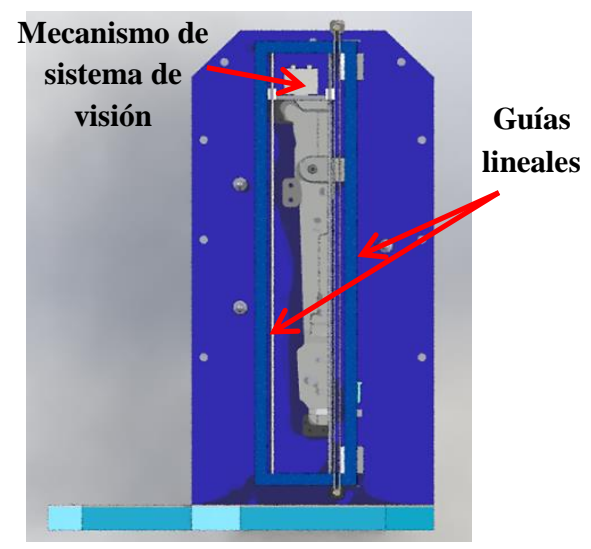

Figura 6 Gage, Checking Fixture con sistema de visión Fuente: Elaboración Propia
En las pruebas físicas se utilizó el software de Matlab para realizar un escaneo mediante barrido vertical para la obtención de imágenes y que mediante una etapa de procesamiento de dichas imágenes, se convierten en parámetros de interés que puedan ser evaluados.

En la figuras 7 y 8 se muestra una fotografía de la primera región de la pieza tomada en el proceso de escaneo y su relación gráfica de las variables que se definen en el dibujo de referencia para identificar variables de diseño en la pieza.

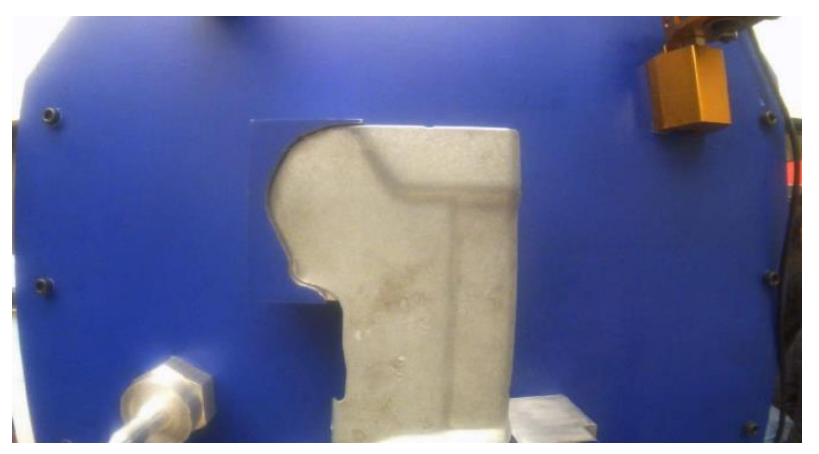

Figura 7 Toma de imagen de la primera región de escaneo Fuente: Elaboración Propia

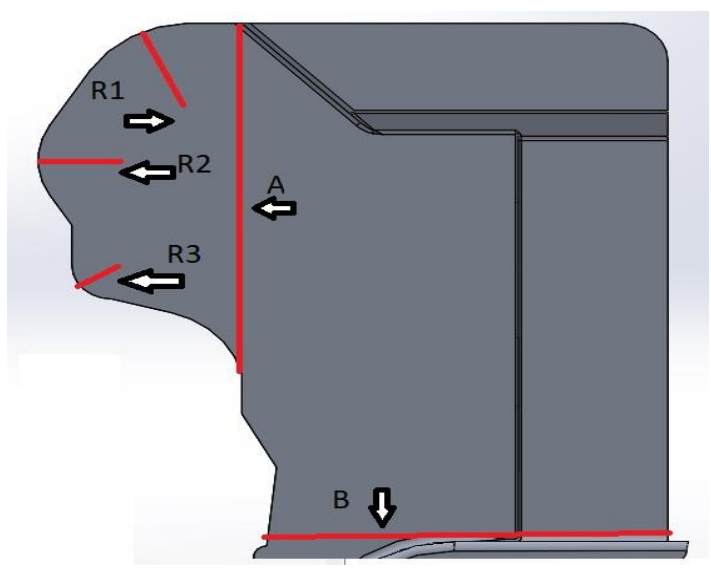

Figura 8 Identificación de los parámetros que se analizan empleando como interfaz el Software de Matlab Fuente: Elaboración Propia

Una vez que se tienen los datos correspondientes es importante seleccionar los gráficos de control adecuados que nos brinden la información confiable de cómo se está comportando la variable al evaluarla en las diferentes tomas de muestras, generando con ello el sistema de control del proceso (SPC). Ver Figura 9. 


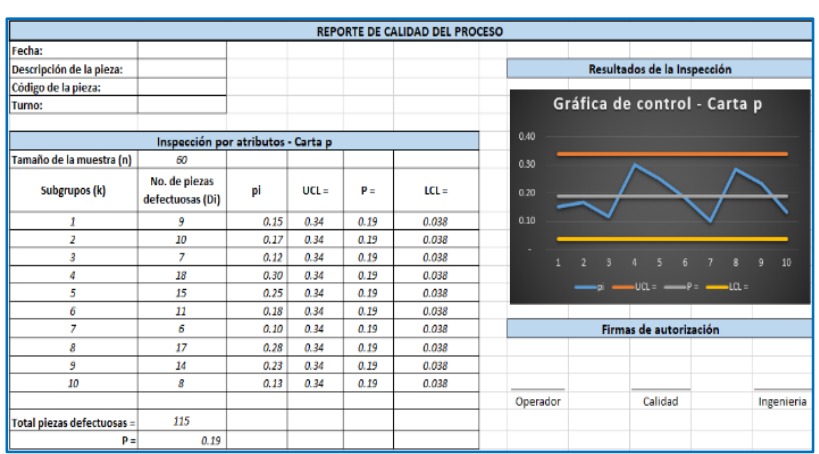

Figura 9 Datos y gráficos de control para evaluar a la variable sujeto de estudio

Fuente: Elaboración Propia

Para validar el dispositivo se lleva a cabo mediante la aplicación del análisis del sistema de medición (MSA) y determinar mediante estudios de R\&R si es confiable el dispositivo.

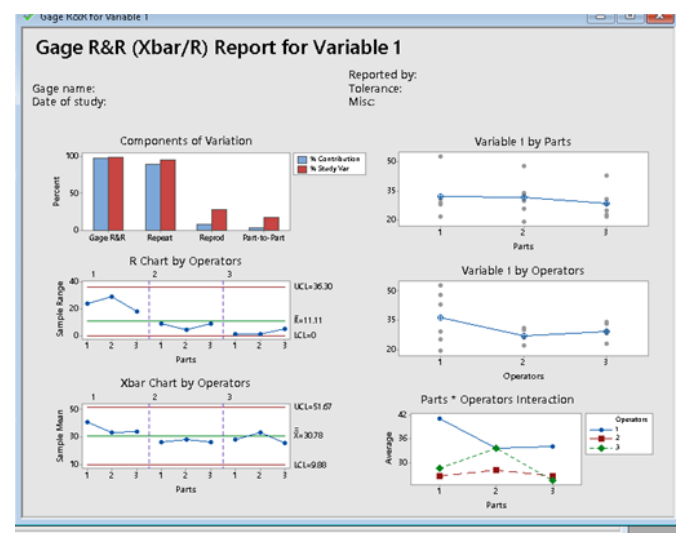

Figura 10 Analisis R\&R para validar el Gage empleando Minitab

Fuente: Elaboración Propia

Por ultimo una vez validado el equipo de medición (gage) este se libera y se puede colocar en la línea de trabajo para llevar a cabo las revisiones en situ y obtener los parámetros correspondientes de una manera automatizada.

\section{Resultados}

Hasta el momento se sigue con la etapa de la construcción del dispositivo de medición, se busca con ello implementar el sistema de visión adecuado y controlar el medio y sus parámetros de operación para evitar interferencias o ruidos que puedan desviar los valores esperados.

Hasta este punto la metodología propuesta, tiene congruencia con el desarrollo del prototipo del Checking Fixture buscando con ello optimizar los tiempos de lecturas de las variables y evitar errores humanos en la medición.
En lo que respecta a la parte de visión se sigue llevando a cabo la programación correspondiente para que se adecue a la pieza a estudiar y establecer una lógica que sirva de plataforma para proyectos futuros.

\section{Conclusión}

El empleo de herramientas de simulación tanto en el diseño como en la manufactura (CAD/CAM) del dispositivo Checking Fixture, han sido de gran utilidad porque ayudan a identificar áreas de oportunidad y establecer estrategias para la fabricación de estos dispositivos de medición.

Se espera que las herramientas que nos hacen falta por aplicar contribuyan de igual manera a robustecer la metodología propuesta en este tema de estudio y que sirva a su vez para que quien desarrolle este tipo de herramentales tenga una orientación respecto a que debe tomar en cuenta para el logro de un dispositivo de medición confiable cumpliendo dos elementos fundamentales que sea preciso y que sea exacto.

\section{Referencias}

Kuigang Yu, ShiyaWang, YananWang and Zhihong Yang (2018). A flexible fixture design method research for similar automotive body parts of different automobiles. Advances in Mechanical Engineering Vol. 10(2) 1-8. SAGE Journals.

Barros Losada, T. (2017). La Industria 4.0: Aplicaciones e Implicaciones. Universidad de Sevilla, Sevilla, España. 2017.

Evans, M. A. (s.f.). The integration of rapid prototyping within Industrial Desing practice. Loughborough University, Reino Unido. 2002.

Gonzalez Gonzalez, C., \& Zeleny Vazquez, J. R. (1995). Metrologia. EDMEX, Mexico: McGraw- Hill Interamericana de Mexico S.A de C.V.

Gutierrez Pulido, H. d. (2013). Control Estadístico de la Calidad y Seis Sigma. DF, Mexico.: McGraw-Hill Interamericana Editores, S.A de C.V.

Jensen, C., D. Helsel, J., \& R. Short, D. (2003). Dibujo y diseño en Ingenieria. McGraw- Hill, Interamericana de Mexico S.A de C.V.

PAZ-CABRERA, Mauro, MANDUJANO-NAVA, Arturo, LANDACALDERON, Rosa María y MENDOZA-DERRAMADERO, José De La Cruz. Diseño y desarrollo de un prototipo Checking - Fixture para componentes de la industria automotriz con un enfoque hacia la industria 4.0. Revista del Desarrollo Tecnológico. 2019 
Groover M., Fundamentos de Manufactura Moderna, 3ra edición, Mc Graw Hill, 2007.

Grupo de Acciones de la Industria Automotriz (AIAG), Análisis de Sistemas de Medición: Manual MSA, 4ta edición, 2010.

H. Roberto Galicia Sánchez, Noé García Lira, Antonio Herrera Martínez, Jorge Mayén González, Mauro Méndez Aranda, Salvador Sánchez González, Metrología Geométrica Dimensional, AGT EDITOR, S.A., 2003.

Paul D. Q. Campbell, "Basic Fixture Design", Industrial Press Inc., 1994.

The American Society of Mechanical Engineers, "Dimensioning and Tolerancing" ASME Y 14.5M USA, 1994. 